\title{
Ameliorative effects and mechanism of crocetin in arsenic trioxide-induced cardiotoxicity in rats
}

\author{
ZHIFENG ZHAO ${ }^{1 *}$, JINGHAN LI ${ }^{2 *}$, BIN ZHENG $^{1}$, YINGRAN LIANG $^{1}$, JING SHI $^{3}$, \\ JIANPING ZHANG ${ }^{4,5}, \mathrm{XUE} \mathrm{HAN}^{1}, \mathrm{LI} \mathrm{CHU}^{1,4}, \mathrm{XI} \mathrm{CHU}^{3}$ and YONGGANG GAO ${ }^{2}$ \\ ${ }^{1}$ Department of Pharmaceutics, School of Pharmacy; ${ }^{2}$ Department of Preventive Medicine, \\ School of Basic Medicine, Hebei University of Chinese Medicine, Shijiazhuang, Hebei 050200; \\ ${ }^{3}$ Department of Pharmacy, The Fourth Hospital of Hebei Medical University, Shijiazhuang, Hebei 050011; \\ ${ }^{4}$ Hebei Key Laboratory of Integrative Medicine on Liver-Kidney Patterns; ${ }^{5}$ Department of Pharmacology \\ School of Basic Medicine, Hebei University of Chinese Medicine, Shijiazhuang, Hebei 050200, P.R. China
}

Received April 12, 2020; Accepted August 20, 2020

DOI: $10.3892 / \mathrm{mmr} .2020 .11587$

\begin{abstract}
Arsenic trioxide (ATO) is commonly used to treat patients with acute promyelocytic leukemia since it was authorized by the U.S. Food and Drug Administration in the 1970s, but its applicability has been limited by its cardiotoxic effects. Therefore, the aim of the present study was to investigate the cardioprotective effects and underlying mechanism of crocetin (CRT), the critical ingredient of saffron. Sprague-Dawley rats were then randomly divided into four groups (n=10/group): i) Control group; ii) ATO group, iii) CRT-low $(20 \mathrm{mg} / \mathrm{kg})$ group; and iv) CRT-high ( $40 \mathrm{mg} / \mathrm{kg}$ ) group. Rats in the Control and ATO groups were intraperitoneally injected with equal volumes of $0.9 \%$ sodium chloride solution, and CRT groups were administered with either 20 and $40 \mathrm{mg} / \mathrm{kg}$ CRT. Following $6 \mathrm{~h}$, all groups except the Control group were intraperitoneally injected with $5 \mathrm{mg} / \mathrm{kg}$ ATO over 10 days. Cardiotoxicity was indicated by changes in electrocardiographic (ECG) patterns,
\end{abstract}

Correspondence to: Dr Xi Chu, Department of Pharmacy, The Fourth Hospital of Hebei Medical University, 12 Jiankang Road, Shijiazhuang, Hebei 050011, P.R. China

E-mail: chux2014@126.com

Professor Yonggang Gao, Department of Preventive Medicine, School of Basic Medicine, Hebei University of Chinese Medicine, 6 Xingyuan Road, Shijiazhuang, Hebei 050200, P.R. China

E-mail: gyg3177@163.com

*Contributed equally

Abbreviations: ATO, arsenic trioxide; CAT, catalase; CK, creatine kinase; Con, control group; CRT, crocetin; CRT-L, ATO + low concentration of CRT treatment group; CRT-H, ATO + high concentration of CRT treatment group; ECG, electrocardiogram; GSH-Px, glutathione peroxidase; H\&E, hematoxylin and eosin; LDH, lactate dehydrogenase; MDA, malondialdehyde; LPO, lipid peroxidation; ROS, reactive oxygen species; SirT1, silent information regulator of transcription 1 ; SOD, superoxide dismutase

Key words: CRT, ATO, cardiotoxicity, myocardial protection morphology and marker enzymes. Histomorphological changes in the heart tissue were observed by pathological staining. The levels of superoxide dismutase, glutathione peroxidase, malondialdehyde and catalase in the serum were analyzed using colometric commercial assay kits, and the levels of reactive oxygen species in the heart tissue were detected using the fluorescent probe dihydroethidium. The expression levels of inflammatory factors and activities of apoptosis-related proteins were analyzed using immunohistochemistry. The protein expression levels of silent information regulator of transcription 1 were measured using western blotting. Cardiotoxicity was induced in male Sprague-Dawley rats with ATO (5 mg/kg). CRT (20 and $40 \mathrm{mg} / \mathrm{kg}$ ) and ATO were co-administered to evaluate possible cardioprotective effects. CRT significantly reduced the heart rate and J-point elevation induced by ATO in rats. Histological changes were evaluated via hematoxylin and eosin staining. CRT decreased the levels of creatine kinase and lactate dehydrogenase, increased the activities of superoxide dismutase, glutathione-peroxidase and catalase, and decreased the levels of malondialdehyde and reactive oxygen species. Moreover, CRT downregulated the expression levels of the pro-inflammatory factors IL-1, TNF- $\alpha$, IL-6, Bax and p65, as well as increased the expression of Bcl-2. It was also identified that CRT enhanced silent information regulator of transcription 1 protein expression. Thus, the present study demonstrated that CRT treatment effectively ameliorated ATO-induced cardiotoxicity. The protective effects of CRT can be attributed to the inhibition of oxidative stress, inflammation and apoptosis. Therefore, CRT represents a promising therapeutic method for improving the cardiotoxic side effects caused by ATO treatment, and additional clinical applications are possible, but warrant further investigation.

\section{Introduction}

Arsenic trioxide (ATO) is the highly toxic main ingredient of arsenic, which is used in Chinese medicine. For instance, ATO has been used to successfully treat recurrent and refractory acute promyelocytic leukemia (APL) since the 1970s (1). The efficacy of ATO is explained by its ability 
to induce apoptosis and the partial differentiation of APL cells (2), and understanding the mechanism of ATO against APL has enabled additional breakthroughs in cancer treatment (2). Although well-established for its therapeutic effects, QT interval (measure of the time between the start of the $\mathrm{Q}$ wave and the end of the $\mathrm{T}$ wave in the heart's electrical cycle) prolongation, action potential variation, torsade de pointes and sudden cardiac death are associated with ATO treatment and have hindered its application $(3,4)$. The major cause of cardiotoxicity is the increased oxidative stress resulting from arsenic deposition (5), and this likely involves a variety of mechanisms, including oxidative DNA damage, the production of reactive oxygen species (ROS) in cardiomyocytes and arsenic accumulation (6). Oxidative stress is the most compelling of the numerous mechanisms underlying ATO-induced toxicity, as it is a key driver in apoptosis and myocardial injury $(7,8)$. Therefore, antioxidative agents may protect against ATO-induced cardiotoxicity. For example, the antioxidant resveratrol protects against ATO-induced cardiotoxicity by reducing oxidative stress (7).

The accumulation of ROS causes oxidative damage due to an imbalance in the production and elimination of oxygen free radicals in cells (9). Free radicals can induce a series of oxidation reactions that cause damage from the molecular to the organ level (10). Malondialdehyde (MDA), a product of lipid peroxidation (LPO), can be used to reflect free radical metabolism and the state of cells attacked by free radicals (11). Superoxide dismutase (SOD) is an oxygen free radical scavenger that protects cells by neutralizing ROS, and SOD levels indirectly represent the ability of the cell to protect itself from free radicals (12). When the production and neutralization of free radicals are in dynamic equilibrium, tissue is not susceptible to damage (13). LPO is caused by the combined effect of unsaturated fatty acids and free radicals, resulting in an imbalance between the unsaturated fatty acids and proteins in membranes (14). This imbalance reduces membrane fluidity and permeability, increases the influx of $\mathrm{Ca}^{2+}$ and indirectly restricts the action of membrane proteins, which leads to cellular swelling and $\mathrm{Ca}^{2+}$ overload (15).

Cytokines are inflammatory mediators involved in immune reactions and pro-inflammatory cytokines, such as IL-1, IL-6 and TNF- $\alpha$, contribute to the pathology and progression of numerous diseases, such as numerous types of inflammatory disease and cancer (16). NF- $\mathrm{kB} / \mathrm{p} 65$ regulates genes involved in inflammatory responses and can be induced in multiple cell types (17). Moreover, arsenic exposure likely increases the expression of inflammation molecules, and as a transcriptional master regulator of pro-inflammatory cytokines, NF- $\mathrm{kB} / \mathrm{p} 65$ can be stimulated by ATO and trigger inflammatory responses (18).

The primary mechanism underlying the adverse effects of arsenic-induced cardiac injury may be associated with the generation of ROS and ROS-induced apoptosis $(19,20)$. Oxidative stress is an important element of ATO-induced mitochondria-mediated apoptosis (21). Apoptosis is a common mechanism of cardiotoxicity, and ATO triggers apoptosis in cells (22). However, clinically suitable, cardioprotective therapeutics do not protect against arsenic toxicity.

Silent information regulator of transcription (SirT1) is a $\mathrm{NAD}^{+}$-dependent histone deacetylase, which regulates the proliferation, apoptosis, differentiation, aging and metabolism of cells in a tissue-specific manner, as well as serves an important role in the modulation of angiocardiopathy (23). The overexpression of SirT1, within a certain range, counteracts myocardial hypertrophy and aging (24). Furthermore, SirT1 exerts pro-survival effects in a variety of cells and tissues due to its antioxidative and anti-apoptotic properties (25), suggesting that SirT1 is a promising mediator of the cardioprotective effects required to combat ATO-induced cardiotoxicity.

Crocetin (CRT; $\mathrm{C}_{20} \mathrm{H}_{24} \mathrm{O}_{4}$; molecular weight, 328; Fig. 1) is the critical ingredient of saffron (Crocus sativus L) and possesses beneficial pharmacological effects (26). In Ayurvedic and other folk medicines, saffron is used for its numerous pharmacological properties such as analgesia, prostration, renoprotection, memory enhancement and anti-depression $(27,28)$. CRT can also reduce LPO in ischemia-reperfusion-induced oxidative damage in male albino rats and scavenge free radicals (28). CRT is a natural carotenoid dicarboxylic acid (29), which possesses a diterpene and has a symmetrical structure with alternating trans-double bonds in alkyl chains and four methyl and carboxyl groups at both ends of the backbone $(30,31)$. CRT has beneficial cardiovascular effects, such as reducing oxidative stress (32) and inhibiting the development of insulin resistance (33), atherosclerosis (34), hypertension (35) and cardiac hypertrophy $(36,37)$. CRT also possesses a broad diversity of pharmacological properties, including antioxidative and anti-inflammatory effects $(38,39)$. CRT significantly decreases LPO, enhances glutathione peroxidase (GSH-Px) and SOD activity and improves the histopathology of the myocardium in hypertrophic tissue (32). Moreover, CRT regulates myocardial enzymes to reduce cardiac cytotoxicity and apoptosis levels $(32,40)$. However, it is yet to be elucidated whether CRT attenuates ATO-induced cardiotoxicity and reduces arsenic accumulation.

Based on the aforementioned findings, the present study aimed to investigate the ameliorative effects and mechanism of CRT on ATO-induced cardiotoxicity in rats. It was hypothesized that CRT could protect against cardiotoxicity, and thus an ATO-induced cardiotoxicity model was established to examine CRT. Furthermore, oxidative stress, inflammatory cytokines and apoptotic factors were evaluated in rats with cardiotoxicity.

\section{Materials and methods}

Experimental materials. CRT (98\% purity) was purchased from Shanghai Yuanye Bio-Technology Co., Ltd. ATO was purchased from Beijing Shuanglu Pharmaceutical Co., Ltd. Sodium chloride solution was purchased from Shijiazhuang No. 4 Pharmaceutical Co., Ltd. CRT and ATO were dissolved in saline and used immediately after preparation. CRT weas dissolved to a final concentration of 20 or $40 \mathrm{mg} / \mathrm{kg}$, while ATO was dissolved to a final concentration of $5 \mathrm{mg} / \mathrm{kg}$.

Experimental animals. In total, 40 adult male Sprague-Dawley (SD) rats (age, 6-8 weeks; weight 180-220 g) were obtained from the Laboratory Animal Center of Hebei Medical University. Rats were raised in rust-free cages at $22-25^{\circ} \mathrm{C}$ and $45-60 \%$ relative humidity on a 12 -h light-dark cycle with ad libitum intake of granular rat chow and tap water. Veterinarians 


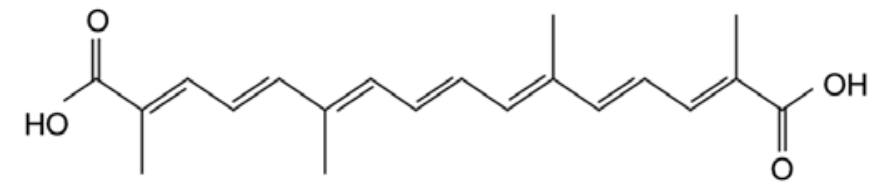

Figure 1. Chemical structure of crocetin.

and researchers monitored the animals' health and behavior twice per day at 8:00 and 17:00. All animal experiments were approved by the Institutional Animal Experimental Ethics Committee of the Hebei University of Chinese Medicine.

Experimental model. After 7 days of adaptive feeding, the rats were randomly separated into four groups $(\mathrm{n}=10)$ : Control (Con), ATO, CRT-L (ATO + low concentration of CRT treatment) and CRT-H (ATO + high concentration of CRT treatment). The CRT-L and CRT-H groups were given 20 and $40 \mathrm{mg} / \mathrm{kg}$ CRT, respectively, followed by $5 \mathrm{mg} / \mathrm{kg}$ ATO $6 \mathrm{~h}$ later. The ATO group was given a CRT-matched amount of $0.9 \%$ sodium chloride solution followed by $5 \mathrm{mg} / \mathrm{kg}$ of ATO $6 \mathrm{~h}$ later. The Con group was given the same amount of $0.9 \%$ sodium chloride solution followed by an ATO-matched amount of $0.9 \%$ sodium chloride solution $6 \mathrm{~h}$ later. All treatments were delivered daily via intraperitoneal injection for 10 days. After 10 days, the rats were anesthetized with $1.0 \mathrm{~g} / \mathrm{kg}$ ethyl carbamate intraperitoneally $(41,42)$. An electrocardiogram (ECG) was performed in anesthetized rats. Then, the blood $(5 \mathrm{ml})$ was collected by exsanguination from the abdominal aorta (43), and the serum was isolated via centrifugation at $1,500 \mathrm{x} \mathrm{g}$ for $10 \mathrm{~min}$ at $4^{\circ} \mathrm{C}$ and stored at $-20^{\circ} \mathrm{C}$ for further analyses.

The rats were euthanized with intraperitoneal injection of sodium pentobarbital (200 mg/kg) (44). Then, hearts were quickly dissected out from each animal and the blood was removed using cold physiological saline. The heart tissue samples were fixed with $4 \%$ paraformaldehyde solution at room temperature for $24 \mathrm{~h}$ or frozen in liquid nitrogen, and then stored at $-20^{\circ} \mathrm{C}$ until use.

Electrocardiogram measurement. After the final treatment, the rats were deprived of food and water for $12 \mathrm{~h}$. The rats were anesthetized, and then three needle electrodes were attached to the right arm, left arm and left leg of the rats (45), and the ECG patterns were recorded using a RM6240BD Biological Signal Collection system (Cheng Yi) to monitor changes in ECG heart rate and J-point elevation.

Histopathological analysis. After rats were euthanized via overdose sodium pentobarbital $(200 \mathrm{mg} / \mathrm{kg})$, the hearts were rapidly excised. Prior to hematoxylin and eosin $(\mathrm{H} \& \mathrm{E})$ staining, the apical myocardium was fixed in $4 \%$ paraformaldehyde for $24 \mathrm{~h}$ at room temperature. The heart tissues were removed from the fixing fluid, and were then cleared, dehydrated, macerated and embedded in paraffin. The tissue samples were sectioned at $5-\mu \mathrm{m}$ thickness and were stained with hematoxylin for $15 \mathrm{~min}$ and eosin for $5 \mathrm{~min}$, both at room temperature according to standard procedures (46). The samples were visualized under an optical microscope at $\mathrm{x} 400$ magnification (Leica DM4000B; Leica Microsystems GmbH).
Detection of lactate dehydrogenase $(\mathrm{LDH})$ and creatine kinase $(C K)$ activities. When the rats were anesthetized, blood $(5 \mathrm{ml})$ was collected by exsanguination from the abdominal aorta and serum was isolated via centrifugation at 1,500 $\mathrm{x}$ for $10 \mathrm{~min}$ at $4^{\circ} \mathrm{C}$. Then, diagnostic markers for CK (cat. no. A032) and LDH (cat. no. A020-1) were measured using commercially available LDH and CK kits (Nanjing Jiancheng Bioengineering Institute) according to the manufacturer's instructions.

Detection of ROS. The frozen cardiac tissues were used to analyze ROS generation reflected in the fluorescence intensity of dihydroethidium (DHE; cat. no. KGAF019; Nanjing KeyGen Biotech Co., Ltd.). After the animals were euthanized by overdose of sodium pentobarbital, the hearts were quickly excised, embedded in the optimal cutting temperature (OCT) compound and flash-frozen in liquid nitrogen. The unfixed frozen samples were sectioned (thickness, $10 \mu \mathrm{m}$ ) at $-20^{\circ} \mathrm{C}(47)$. After fixing with $50 \%$ OTC at $-80^{\circ} \mathrm{C}$ for $2 \mathrm{~h}$, the sections were washed three times with PBS for 5 min each time and DHE $(50 \mu \mathrm{M})$ was applied to each tissue section, which was incubated in a light-protected humidified chamber at $37^{\circ} \mathrm{C}$ for $30 \mathrm{~min}$. Sections were washed three times with PBS for 5 min each time (48). Subsequently, the sections were sealed with neutral balsam (cat. no. 10004160; Sinopharm Chemical Reagent Co., Ltd.), and observed under a fluorescence microscope (magnification, x400; Olympus Corporation) (49). ROS production (red staining) was quantified using Image-Pro Plus 6.0 software (Media Cybernetics, Inc.).

Detection of catalase (CAT), SOD, GSH-Px and MDA. Blood $(5 \mathrm{ml})$ samples were collected from the abdominal aorta. Serum was isolated via centrifugation at $1,500 \mathrm{x}$ for $10 \mathrm{~min}$ at $4^{\circ} \mathrm{C}$ and the enzymatic activities of CAT (cat. no. A007-1), SOD (cat. no. A001-1) and GSH-Px (cat. no. A005-1), as well as the concentration of MDA (cat. no. A003-1) were determined using colorimetric commercially available kits (Nanjing Jiancheng Bioengineering Institute) according to the manufacturer's instructions.

Immunohistochemistry.Immunohistochemistrywasperformed according to the methods previously described (50-52). After the heart of the rat was removed, samples were fixed in $4 \%$ paraformaldehyde for $24 \mathrm{~h}$ at room temperature and embedded in paraffin. Then, the paraffin-embedded tissue sections (4- $\mu \mathrm{m}$ thick) were deparaffinized, rehydrated in a descending series of ethanol (100, 95, 90 and $80 \%)$ and immersed in retrieval solution. Sections were incubated with $3 \%$ methanol- $\mathrm{H}_{2} \mathrm{O}_{2}$ for $20-30 \mathrm{~min}$ at $37^{\circ} \mathrm{C}$ to eliminate endogenous peroxidase activity. Non-specific staining was blocked with $5 \% \mathrm{Ig}$ blocking reagent and 5\% serum (Shanghai Regal Biological Technology Development Co., Ltd.) for $15 \mathrm{~min}$ at $37^{\circ} \mathrm{C}$ after rinsing. Sections were incubated with primary antibodies against IL-1 (1:100; cat. no. 16765-1-AP; ProteinTech Group, Inc.), IL-6 (1:100; cat. no. 21865-1-AP; ProteinTech Group, Inc.), Bax (1:100; cat. no. 50599-2-Ig; ProteinTech Group, Inc.), TNF- $\alpha$ (1:100; cat. no. 60291-1-Ig; ProteinTech Group, Inc.), Bcl-2 (1:100; cat. no. 26593-1-AP; ProteinTech Group, Inc.) and p65 (1:100; cat. no. 10745-1-AP; ProteinTech Group, Inc.) at $4^{\circ} \mathrm{C}$ overnight. The following day, the sections were maintained at room temperature and washed with PBS three 

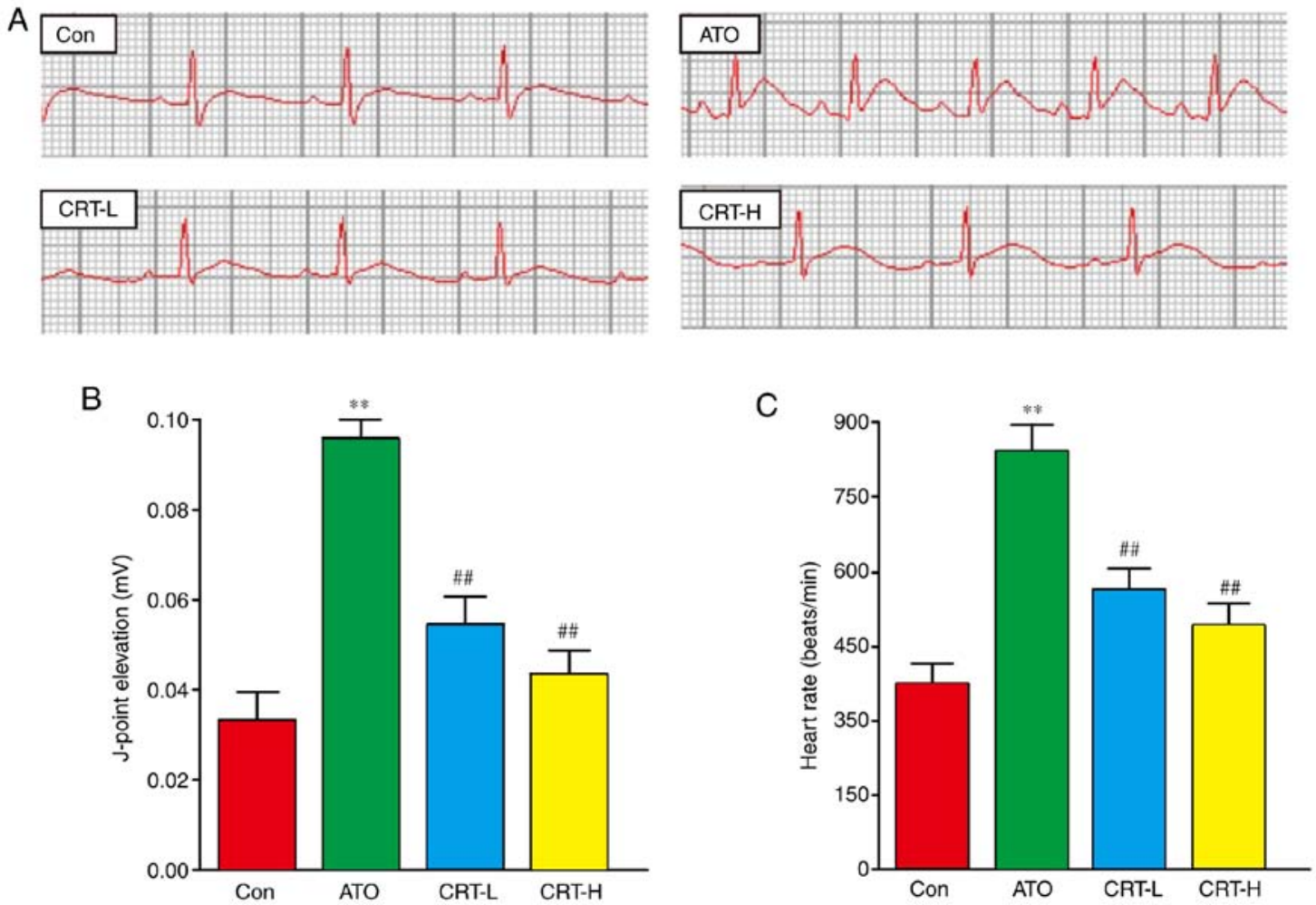

Figure 2. Effects of CRT on ECG. (A) Representative ECG tracings from each group. Statistical analysis of (B) J-point elevation and (C) heart rate. Data are presented as the mean \pm SEM for each group, $n=10 .{ }^{* *} \mathrm{P}<0.01$ vs. Con group; ${ }^{\# \#} \mathrm{P}<0.01$ vs. ATO group. CRT, crocetin; ECG, electrocardiogram; Con, control; ATO, arsenic trioxide; CRT-L, ATO + low concentration of CRT treatment group; CRT-H, ATO + high concentration of CRT treatment group.

times for 5 min each time. Next, the sections were incubated with HRP-conjugated anti-rabbit and anti-mouse secondary antibodies (1:200; cat. nos. PV-6001 and PV-6002; OriGene Technologies, Inc.) at room temperature for $20 \mathrm{~min}$ and then washed in PBS three times (5 min each time). The 3,3'diaminobenzidine (DAB) dye solution was added onto the sections and incubated for 5-10 sec at room temperature, at which point the reaction was stopped using water washes three times. Color development was induced with a DAB kit (cat. no. ZLI-9019; OriGene Technologies, Inc.) and counterstained with hematoxylin for $2 \mathrm{~min}$ at room temperature. The slides were subsequently dehydrated with an ascending gradient ethanol series $(30,50,70,80,90,95$ and $100 \%)$ for 3 min each at room temperature. Xylene was used to make the sections transparent and these were then sealed. The slices were visualized using a digital light microscope (magnification, $x 400$ ) and the resulting images were analyzed using Image-Pro 6.0 software (Media Cybernetics, Inc.).

Western blotting. Frozen heart tissues were removed, homogenized and lysed in ice-cold cell lysis buffer (cat. no. 9803; Cell Signaling Technology, Inc.). The proteins were extracted from different issues and quantified using the BCA assay. Protein samples $(50 \mu \mathrm{g})$ were subjected to SDS-PAGE and transferred to PVDF membranes (EMD Millipore) (51). The membranes were blocked with 5\% skim milk in TBS-0.1\% Tween-20 (TBST) buffer at room temperature for $1 \mathrm{~h}$. The cell membranes were incubated overnight at $4^{\circ} \mathrm{C}$ with primary antibody in blocking buffer, including anti-SirT1 (cat. no. 60303-1-Ig; 1:1,000; ProteinTech Group, Inc.) and anti- $\beta$-actin (cat. no. TA-09;
1:1,000; OriGene Technologies, Inc.). Next, the membranes were washed three times with TBST and then incubated with horseradish peroxidase-conjugated secondary anti-rabbit and anti-mouse antibodies (cat. nos. 7074 and 7076; 1:5,000; Cell Signaling Technology, Inc.) for $1 \mathrm{~h}$ at room temperature. Membranes were washed three times and proteins were visualized using the ECL Detection system (TransGen Biotech Co., Ltd.) (53), and imaged using Tanon-1600 Gel Image Analysis system (Tanon Science and Technology Co., Ltd.). Bands were quantified using Tanon Gis 1D software (Tanon Science and Technology Co., Ltd.) (49).

Statistical analysis. All data were statistically analyzed using SPSS 20.0 software (IBM Corp.) and graphs were created using Origin Pro 9.1 software (Europa Science Ltd.). A single-factor ANOVA was used for multigroup comparisons followed by Tukey's test. Data are presented as the mean \pm SEM. Each experiment was repeated $\geq 3$ times. $\mathrm{P}<0.05$ was considered to indicate a statistically significant difference.

\section{Results}

Effect of CRT on ECG. Sample tracings of ECG from the experimental animals are presented in Fig. 2A. Compared with the Con group, the heart rate and J-point of the rats in the ATO group were significantly increased $(\mathrm{P}<0.01)$, suggesting that the experimental myocardial injury model was successfully established (Fig. 2B and C). Moreover, these two indexes were significantly lower in the CRT-L and CRT-H groups compared with the ATO group $(\mathrm{P}<0.01)$. 

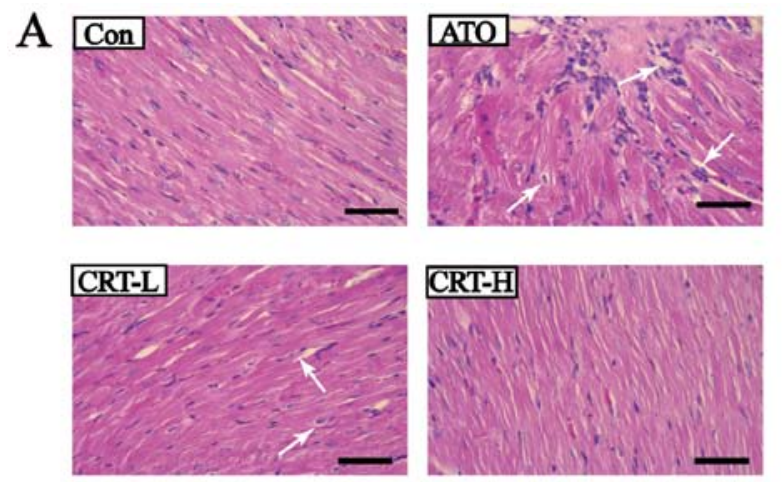

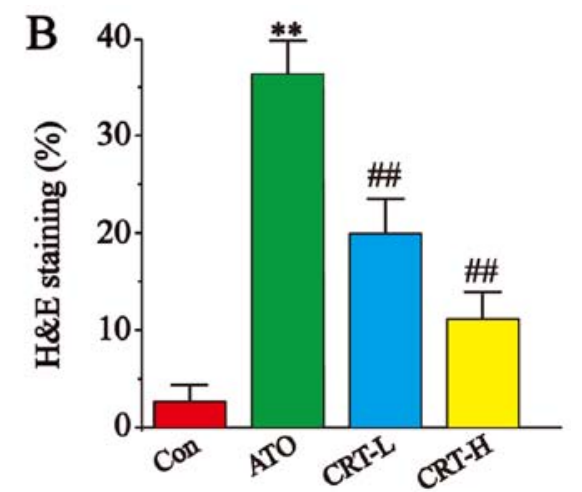

Figure 3. Effects of CRT on H\&E staining in ATO-induced cardiotoxicity in rats. (A) Myocardial tissue obtained from the Con, ATO, CRT-L and CRT-H groups. Histopathological changes are indicated by white arrows. (B) Pathological damage scores of the heart tissue in the Con, ATO, CRT-L and CRT-H groups. Scale bar, $50 \mu \mathrm{m}$. Data are presented as the mean $\pm \mathrm{SEM}$ for each group, $\mathrm{n}=10 .{ }^{* *} \mathrm{P}<0.01 \mathrm{vs}$. Con group; ${ }^{\# \#} \mathrm{P}<0.01$ vs. ATO group. CRT, crocetin; Con, control; ATO, arsenic trioxide; CRT-L, ATO + low concentration of CRT treatment group; CRT-H, ATO + high concentration of CRT treatment group; H\&E, hematoxylin and eosin.
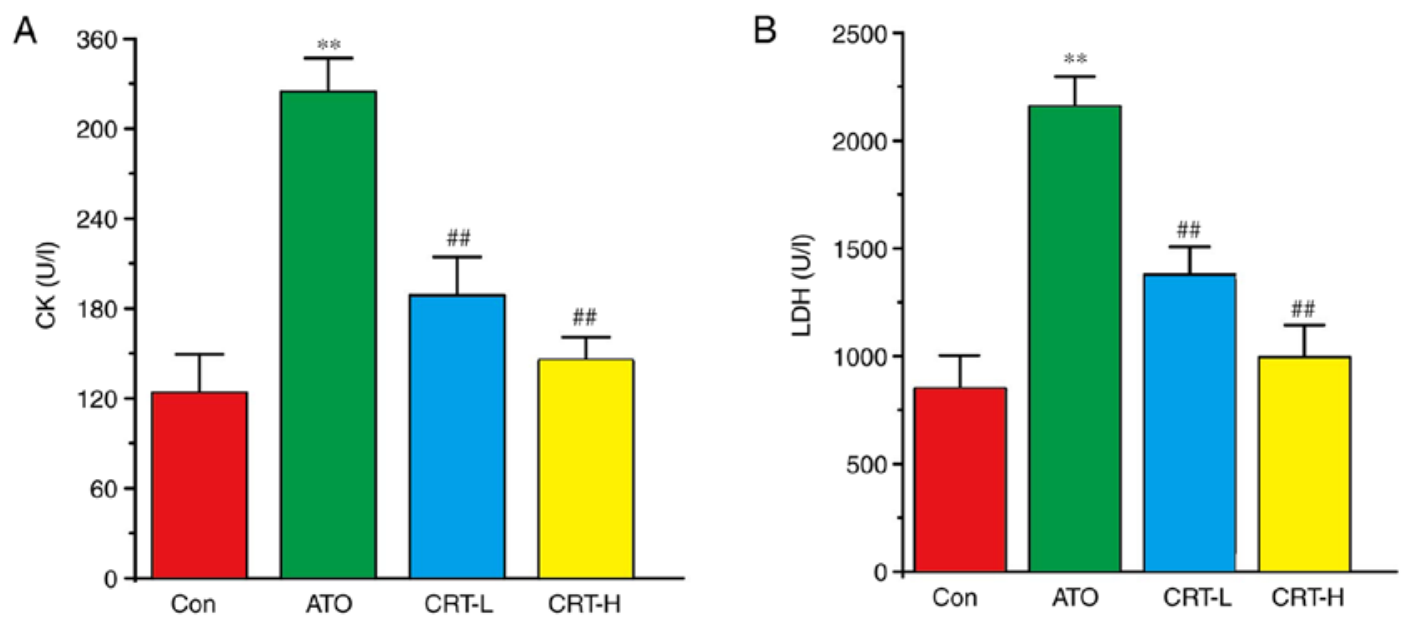

Figure 4. Effects of CRT on CK and LDH activities in ATO-induced cardiotoxicity in rats. Serum was collected from the Con, ATO, CRT-L and CRT-H groups and assayed for (A) CK and (B) LDH enzyme activities using CK and LDH kits. Data are presented as the mean \pm SEM for each group, $n=10 .{ }^{* *} \mathrm{P}<0.01 \mathrm{vs}$. Con group; ${ }^{\# /} \mathrm{P}<0.01$ vs. ATO group. CRT, crocetin; Con, control; ATO, arsenic trioxide; CRT-L, ATO + low concentration of CRT treatment group; CRT-H, ATO + high concentration of CRT treatment group; CK, creatine kinase; LDH, lactate dehydrogenase.

Effects of CRT on histopathology. The results of the histopathological examination of the myocardial tissues are presented in Fig. 3. The H\&E-stained heart sections from the Con group had healthy cardiomyocyte structures. However, the sections from the ATO-induced cardiotoxicity group contained extensive necrosis, inflammatory cell infiltration, cytoplasmic vacuolization, slight fiber swelling, soft interstitial edema and myofiber loss. Compared with the Con group, the ATO caused a significant increase in the morphological changes $(\mathrm{P}<0.01$; Fig. 3B). It was found that treatment with CRT-L and CRT-H significantly improved these signs of ATO-induced cardiotoxicity ( $\mathrm{P}<0.01$; Fig. 3B), suggesting that CRT exerted a potent protective effect in this model of cardiac injury.

Effect of CRT on CK and LDH activities. Serum CK and LDH activities were significantly increased in the ATO group ( $\mathrm{P}<0.01$; Fig. 4A and B) compared with the Con group. Relative to the ATO group, CRT treatment resulted in a significant decrease in CK and LDH activities at $20 \mathrm{mg} / \mathrm{kg} /$ day $(\mathrm{P}<0.01)$ and at $40 \mathrm{mg} / \mathrm{kg} /$ day $(\mathrm{P}<0.01)$.
Effects of CRT on ROS release. A significant increase in ROS generation (red) was observed in the rat hearts from the ATO group in comparison with the Con group (Fig. 5). However, CRT preconditioning partially eliminated these changes $(\mathrm{P}<0.01)$, and ROS generation was markedly lower in the CRT-H group compared with the CRT-L group.

Effects of CRT on SOD, GSH-PX, MDA and CAT. Compared with the Con group, the ATO group had increased myocardial MDA activity $(\mathrm{P}<0.01)$, but decreased SOD, GSH-Px and CAT activities $(\mathrm{P}<0.01)$ (Fig. 6A-D). Furthermore, CRT administration $(20$ and $40 \mathrm{mg} / \mathrm{kg} / \mathrm{day})$ led to a dose-dependent increase in SOD, GSH-Px and CAT activities $(\mathrm{P}<0.01$ or $\mathrm{P}<0.05)$, along with a concomitant decrease in MDA content compared with the ATO group $(\mathrm{P}<0.01$ or $\mathrm{P}<0.05)$. Therefore, $\mathrm{CRT}$ attenuated the oxidative stress associated with ATO-induced cardiotoxicity in a dose-dependent manner $(\mathrm{P}<0.01)$.

Effects of CRT on IL-1, IL- 6 and TNF- $\alpha$ expression levels. The expression levels of IL-1, IL- 6 and TNF- $\alpha$ in cardiac tissue were assessed using immunohistochemistry (Fig. 7A-F). 

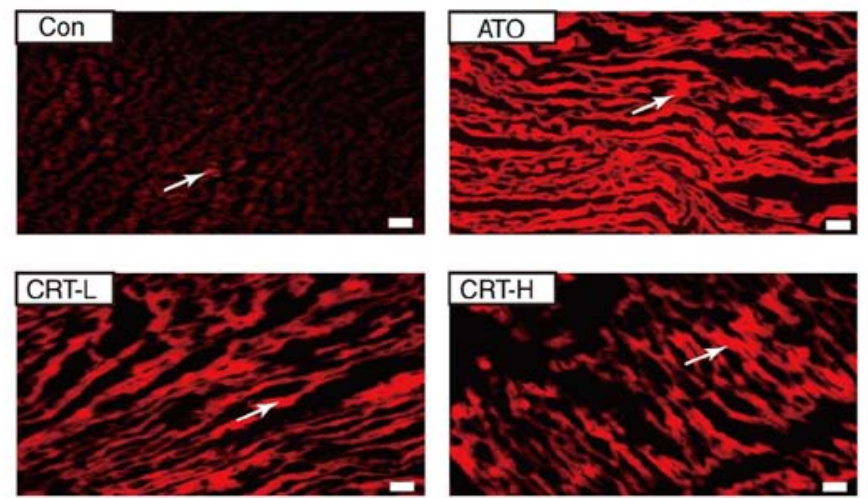

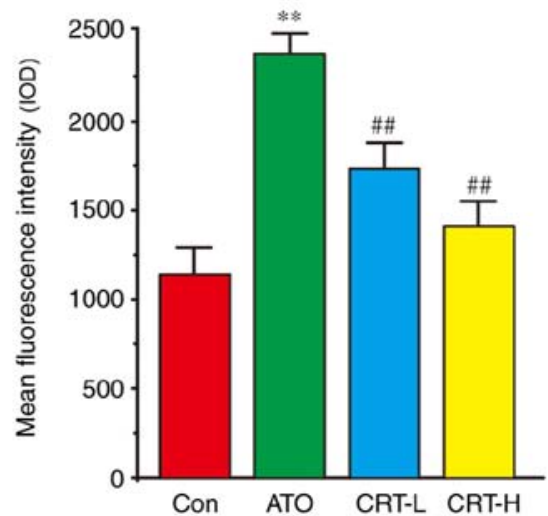

Figure 5. Actions of CRT on ROS in rat heart tissue using the fluorescent probe dihydroethidium. Effect of CRT and ATO on ROS generation in the heart tissue of Con, ATO, CRT-L and CRT-H animals. Areas with ROS generation are indicated by white arrows. Data are presented as the mean \pm SEM for each group $\mathrm{n}=10 .{ }^{* * *} \mathrm{P}<0.01$ vs. Con group; ${ }^{\# \#} \mathrm{P}<0.01$ vs. ATO group. CRT, crocetin; Con, control; ATO, arsenic trioxide; CRT-L, ATO + low concentration of CRT treatment group; CRT-H, ATO + high concentration of CRT treatment group; ROS, reactive oxygen species; IOD.

A

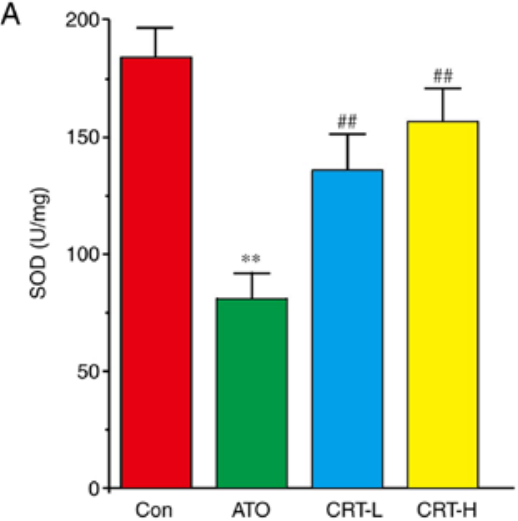

$\mathrm{C}$

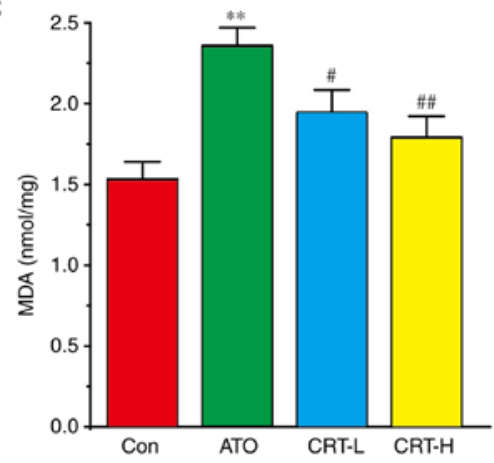

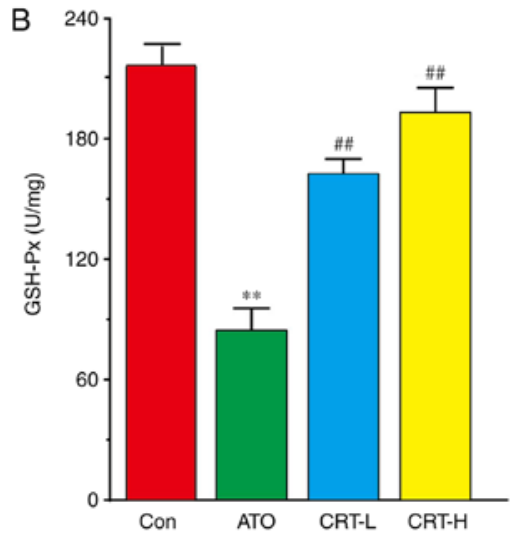

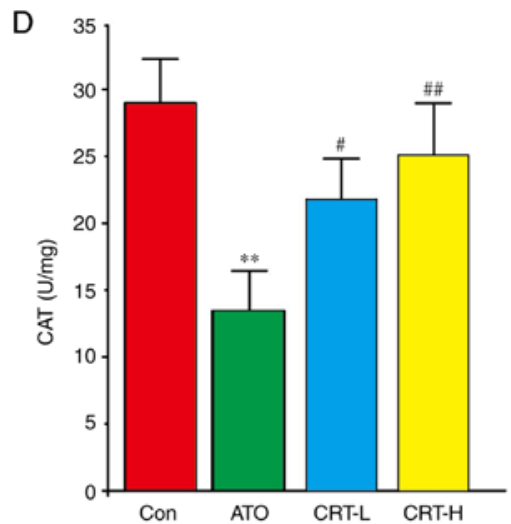

Figure 6. Effects of CRT on SOD, GSH-Px, MDA and CAT levels in ATO-induced cardiotoxicity in rats. Serum was analyzed for (A) SOD, (B) GSH-Px, (C) MDA and (D) CAT in the Con, ATO,CRT-L and CRT-H groups. Data are presented as the mean \pm SEM for each group, $n=10$. ${ }^{* *} \mathrm{P}<0.01$ vs. Con group; ${ }^{*} \mathrm{P}<0.05$, ${ }^{\# \#} \mathrm{P}<0.01$ vs. ATO group. CRT, crocetin; Con, control; ATO, arsenic trioxide; CRT-L, ATO + low concentration of CRT treatment group; CRT-H, ATO + high concentration of CRT treatment group; SOD, superoxide dismutase; GSH-Px, glutathione peroxidase; MDA, malondialdehyde; CAT, catalase.

IL-1, IL-6, and TNF- $\alpha$ expression was low in the Con group (Fig. 7A-F) and increased after ATO treatment $(\mathrm{P}<0.01)$. The immunoreactive area was also more widespread in the ATO group compared with the Con group $(\mathrm{P}<0.01)$. CRT treatment (20 and $40 \mathrm{mg} / \mathrm{kg}$ ) significantly prevented the large increased expression of IL-1, IL-6, and TNF- $\alpha$ and restricted their prevalence. These findings demonstrated that CRT attenuated the expression levels of IL-1, IL- 6 and TNF- $\alpha$ in a dose-dependent manner.
Effects of CRT on $p 65$ expression. The expression of p65 was higher in the ATO group compared with the Con group $(\mathrm{P}<0.01)$, while treatment with CRT decreased p65 expression compared with the ATO group $(\mathrm{P}<0.01)$. These results demonstrated that CRT treatment could prevent the cascade of intracellular signaling (Fig. 8).

Effects of CRT on Bax and Bcl-2 expression. The expression levels of Bax and Bcl-2 were also detected via 
A IL-1
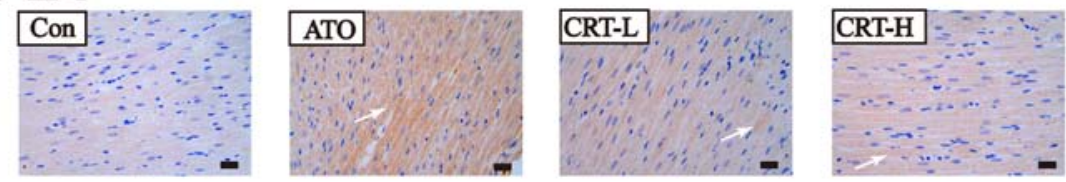

B IL-6
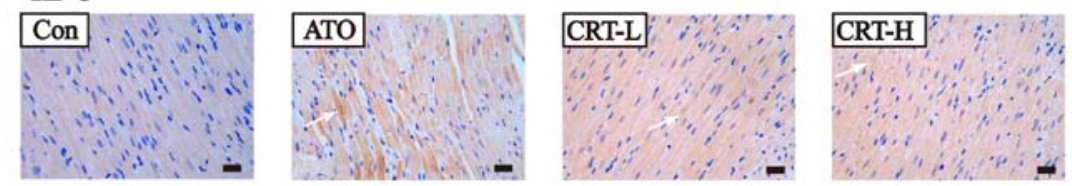

C TNF- $\alpha$
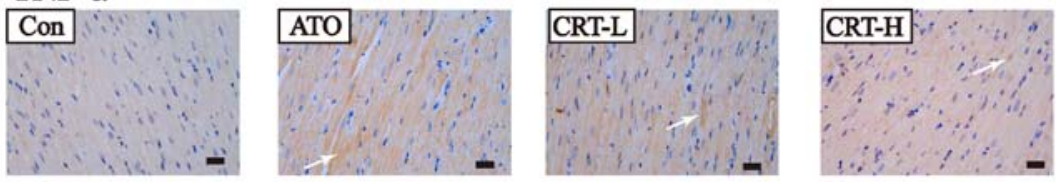

D

E
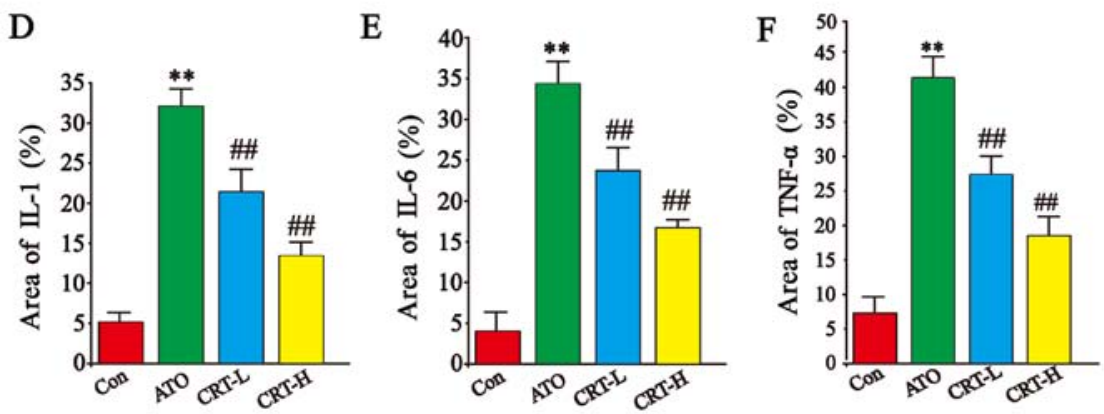

Figure 7. Effects of CRT on the expression levels of IL-1, IL-6 and TNF- $\alpha$ in ATO-treated rats detected via immunohistochemistry. Anatomical location of (A) IL-1 expression, (B) IL-6 expression and (C) TNF- $\alpha$ expression. Immunoreactive IL-1, IL-6 and TNF- $\alpha$ areas are indicated by white arrows. Quantification of the \% area of (D) IL-1 expression, (E) IL-6 expression and (F) TNF- $\alpha$ expression. Scale bar, $50 \mu \mathrm{m}$; magnification, x400. Data are presented as the mean \pm SEM for each group, $n=10 .{ }^{* *} \mathrm{P}<0.01$ vs. Con group; ${ }^{\# \#} \mathrm{P}<0.01$ vs. ATO group. CRT, crocetin; Con, control; ATO, arsenic trioxide; CRT-L, ATO + low concentration of CRT treatment group; CRT-H, ATO + high concentration of CRT treatment group.
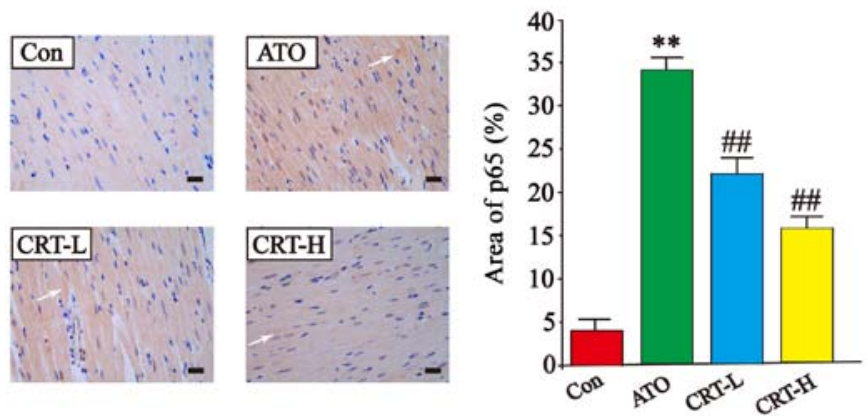

Figure 8. Effects of CRT on the expression of p65 in ATO-induced cardiotoxicity in rats as detected via immunohistochemistry. Morphological location and percent area of p65 expression. p65-immunoreactive areas are indicated by white arrows. Scale bar, $50 \mu \mathrm{m}$; magnification, $\mathrm{x} 400$. Data are presented as the mean $\pm \mathrm{SEM}$ for each group, $\mathrm{n}=10 .{ }^{* * *} \mathrm{P}<0.01$ vs. Con group; ${ }^{\# \#} \mathrm{P}<0.01$ vs. ATO group. CRT, crocetin; Con, control; ATO, arsenic trioxide; CRT-L, $\mathrm{ATO}+$ low concentration of CRT treatment group; CRT-H, ATO + high concentration of CRT treatment group.

immunohistochemistry of cardiac tissue (Fig. 9A-D). The Bax-immunoreactive area (Fig. 9A) was increased and Bcl-2-immunoreactive area (Fig. 9B) was decreased in ATO-treated sections compared with Con group $(\mathrm{P}<0.01$; Fig. $9 \mathrm{C}$ and D). However, treatment with CRT $(20$ and $40 \mathrm{mg} / \mathrm{kg})$ suppressed Bax upregulation $(\mathrm{P}<0.01)$ and increased $\mathrm{Bcl}-2$ expression $(\mathrm{P}<0.01)$ compared with the ATO group. Thus, it was indicated that CRT reduced the expression of Bax and increased Bcl-2 expression.

Effects of CRT on SirT1 expression. The effects of CRT on SirT1 protein expression was evaluated via western blot analysis (Fig. 10A). The ATO group had decreased SirT1 expression compared with the Con group ( $\mathrm{P}<0.01$; Fig. 10B). Furthermore, SirT1 expression was significantly increased in the CRT-L and CRT-H groups relative to the ATO group. It was demonstrated that CRT treatment caused dose-dependent increases in SirT1 expression. Collectively, these results indicated that SirT1 may be involved in ATO-induced cardiotoxicity and that the protective effects of CRT could be mediated by SirT1.

\section{Discussion}

ATO has a beneficial effect on recurrent and refractory APL (54). However, ATO causes cardiotoxicity, characterized by a drop in antioxidant enzymes that serve as makers of ATO-induced myocardial injury and necrosis (7). Moreover, oxidative insults occur in the hearts of SD rats due to increased formation of ROS, leading to severe histological alterations, including cardiomyocyte necrosis and myocardial hemorrhage (55). In the initiation and succession of ATO-induced myocardial injury, the overproduction of ROS and subsequent 
A Bax

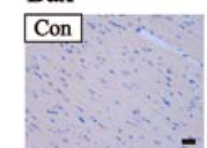

B $\mathrm{Bcl}-2$

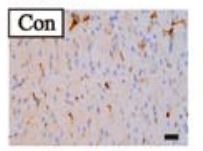

C

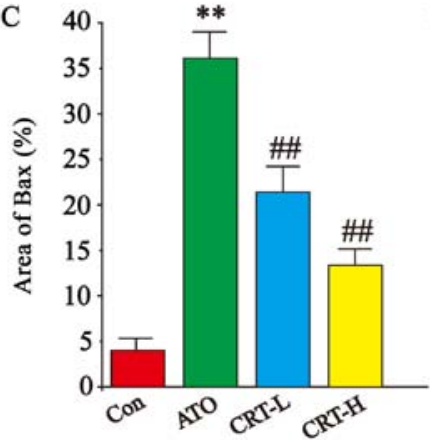

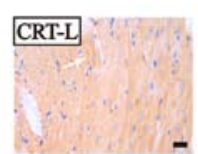

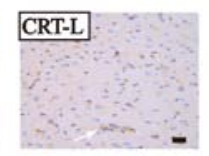

D

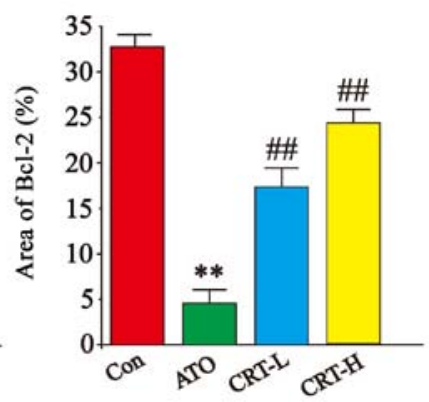

Figure 9. Effects of CRT on the expression levels of Bax and Bcl-2 in ATO-induced cardiotoxicity in rats as detected via immunohistochemistry. Morphological location of (A) Bax expression and (B) Bcl-2 expression. Immunoreactive $\mathrm{Bax}$ and $\mathrm{Bcl}-2$ areas are indicated by white arrows. Quantification of the \% area of (C) Bax expression and (D) Bcl-2 expression. Scale bar, $50 \mu \mathrm{m}$; magnification, $\mathrm{x} 400$. Data are presented as the mean $\pm \mathrm{SEM}$ for each group, $\mathrm{n}=10 .{ }^{* *} \mathrm{P}<0.01$ vs. Con group; ${ }^{\# \#} \mathrm{P}<0.01$ vs. ATO group. CRT, crocetin; Con, control; ATO, arsenic trioxide; CRT-L, ATO + low concentration of CRT treatment group; CRT-H, ATO + high concentration of CRT treatment group.

oxidative stress exerts a crucial role (3). However, the cardiovascular effects of CRT dampen oxidative stress and inhibit the development of insulin resistance, atherosclerosis, hypertension, cardiac hypertrophy and other related diseases (56). The present results suggested that CRT attenuates ATO-induced cardiotoxicity and reduces arsenic accumulation.

CRT is a short carbon chain carotenoid (apocarotenoid of $\mathrm{C}_{20}$ ) with a carboxyl group at each end (Fig. 1). CRT has a potential therapeutic benefit, including its cardioprotective, hepatoprotective, neuroprotective, memory-enhancing, anti-inflammatory, anti-depressant and anxiolytic effects $(57,58)$. Pharmacokinetic studies have reported that after oral administration, CRT is rapidly absorbed by intestinal cells via passive diffusion (29,59-61) and is then distributed into the liver, heart, lungs, kidneys, spleen, adipose tissue and central nervous system $(59,61)$. This absorbed CRT is present in an intact form, and as monoglucuronide and diglucuronide conjugates in plasma (61), and the half-life of CRT is 2.5-2.9 $\mathrm{h}(62)$. No serious adverse events have observed in concentrations up to $40 \mathrm{mg} / \mathrm{kg}$ of orally administered CRT in rat pharmacokinetic experiments (62). The doses used in the present study are based on previous experiments $(62,63)$, and no toxic side effects were evident in the current experiments.

ECG is a sensitive method for detecting cardiac abnormalities and identifying altered cardiac electrical activity (64). An increase in J-point and heart rate was observed in ATO-treated rats in the present study, which may be due to ATO-induced myocardial injury; the increase in J-point and heart rate was decreased in the CRT group, indicative of its protective effect.

A
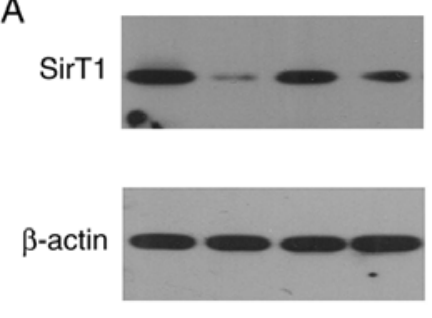

Figure 10. Effects of CRT on SirT1 expression in ATO-induced cardiotoxicity in rats. (A) Protein expression of SirT1 was measured using western blotting. (B) Relative intensities of SirT1 were analyzed after normalizing to $\beta$-actin. The myocardial tissues were obtained from the Con, ATO, CRT-L and CRT-H groups. Data are presented as the mean \pm SEM for each group, $\mathrm{n}=10 .{ }^{* *} \mathrm{P}<0.01$ vs. Con group; ${ }^{\#} \mathrm{P}<0.01$ vs. ATO group. CRT, crocetin; Con, control; ATO, arsenic trioxide; CRT-L, ATO + low concentration of CRT treatment group; CRT-H, ATO + high concentration of CRT treatment group; SirT1, silent information regulator of transcription 1.

In the present study, ATO treatment caused CK and LDH activities to increase, which is indicative of disrupted cardiomyocyte structure and damaged cell membranes $(64,65)$. Thus, the present data suggested that CRT treatment (20 and $40 \mathrm{mg} / \mathrm{kg}$ ) preserved the integrity and functionality of cardiomyocytes.

Arsenic cardiotoxicity affects several physiological processes including cardiac repolarization, intracellular $\mathrm{Ca}^{2+}$ overload, increased $\mathrm{Ca}^{2+}$ currents and elevated intracellular ROS $(66,67)$. Exposure to inorganic arsenic leads to cellular oxidative stress by generating ROS (68). Cardiovascular diseases such as ischemia-reperfusion injury, endothelial dysfunction and atherosclerosis are associated with the release of intracellular ROS $(69,70)$. In the present study, the effects of cardiovascular treatments were assessed in rats using an ATO-induced cardiotoxicity model.

Accumulating evidence suggests that oxidative stress serves an important role in ATO-induced cardiac damage (71). MDA, a typical product of LPO, is formed by the oxidation of polyunsaturated fatty acids caused by ROS and serves as a directional biomarker of oxidative stress that indirectly reflects the degree of cardiomyocyte damage (72). In the present study, ATO raised MDA levels in the myocardial tissue, while CRT protected against arsenic cardiotoxicity, possibly by inhibiting LPO in cardiac tissue. GSH-Px is the major endogenous antioxidative in the body, and its decrease can cause significant damage to cells $(73,74)$. SOD and CAT are antioxidant enzymes whose reinforced activity inhibits oxidative stress and delays the succession of the ATO-induced cardiotoxicity mediated decline in arsenic accumulation (73). Furthermore, CAT, SOD and GSH-Px serve a key role in the regulation of and response to intracellular oxidation (75). In the present study, treatment with CRT increased the levels of CAT, SOD and GSH-Px in myocardial tissue. Thus, it was suggested that CRT relieved ATO-induced oxidative stress, indicated by increased CAT, SOD and GSH-Px activities and simultaneously decreased MDA levels.

In the present study, the mechanism underlying the myocardial protection effect of CRT was investigated by analyzing the 
expression levels of pro-inflammatory markers. ATO induced a significant increase in myocardial TNF- $\alpha$ expression, which was larger compared with the increase observed in IL-1 and IL-6 expression levels. Moreover, ATO induced an increase in p65 expression. Therefore, the anti-inflammatory activity of CRT was demonstrated by its ability to reduce IL-1, IL-6, TNF- $\alpha$ and p65 expression levels, which is consistent with previous studies that reported elevated Bax and reduced Bcl-2 expression after ATO treatment (76-78).

The increased expression of Bax in the ATO group and simultaneous decrease in Bcl-2 expression indicated that apoptosis occurred in the current rat model of cardiotoxicity. Furthermore, CRT decreased the number of apoptotic cells, illustrated here as changes to these apoptosis-related transcripts. These findings are consistent with another previous study (79).

In the present study, western blotting results identified that SirT1 protein expression was decreased in ATO-induced myocardial tissue. However, this lower expression was increased after CRT treatment, which concurs with the results of previous studies, whereby cardiotoxicity was ameliorated through increasing the SirT1 protein expression levels $(80,81)$. Taken together, the present results suggested that treatment with CRT enhanced SirT1 activity, thus counteracting the cardiomyocyte apoptosis caused by ATO.

Several previous studies have demonstrated that ATO could induce morphological changes in the myocardium, such as necrosis, swelling and edema $(55,82)$. The morphological alterations to the myocardium of ATO-treated rats were significantly ameliorated by CRT administration. The present histopathological observations provided evidence of the protective effect of CRT against ATO-induced cardiomyocyte necrosis. It was identified that CRT pretreatment prior to ATO administration efficiently prevented histopathological alterations, which would be particularly beneficial against chemotherapy-induced cardiotoxicity.

In conclusion, the present results suggested that CRT protected cardiomyocytes from ATO-induced cardiotoxicity. The cardioprotective effect of CRT is attributed to the reduction of myocardial oxidative stress, inhibition of inflammation and suppression of apoptosis. Collectively, the present results indicated that pretreatment with CRT ameliorated ATO-induced myocardial damage. Therefore, CRT may have extensive clinical application value in treating ATO-induced cardiotoxicity.

\section{Acknowledgements}

Not applicable.

\section{Funding}

The present stuy was supported by the Research Foundation of Administration of Traditional Chinese Medicine of Hebei Province, China (grant no. 2020188).

\section{Availability of data and materials}

The datasets used and/or analyzed during the current study are available from the corresponding author on reasonable request.

\section{Authors' contributions}

$\mathrm{XC}, \mathrm{YG}, \mathrm{LC}$ and $\mathrm{ZZ}$ designed the study and conducted the experiments. BZ, JL and JS performed the formal experiment and acquired the data. BZ and YL analyzed the data. ZZ, XH and $\mathrm{JZ}$ interpreted the data. $\mathrm{ZZ}$ and JL drafted the manuscript. JL, LC and XH revised the manuscript. All authors read and approved the final manuscript.

\section{Ethics approval and consent to participate}

All animal experiments were approved by the Institutional Animal Experimental Ethics Committee of Hebei University of Chinese Medicine. All experimental procedures complied with the protocols and ethical regulations provided by the Institutional Animal Experimental Ethics Committee of Hebei University of Chinese Medicine.

\section{Patient consent for publication}

Not applicable.

\section{Competing interests}

The authors declare that they have no competing interests.

\section{References}

1. Rao Y, Li R and Zhang D: A drug from poison: How the therapeutic effect of arsenic trioxide on acute promyelocytic leukemia was discovered. Sci China Life Sci 56: 495-502, 2013.

2. Sfaxi I, Charradi K, Limam F, El May MV and Aouani E: Grape seed and skin extract protects against arsenic trioxide induced oxidative stress in rat heart. Can J Physiol Pharmacol 94: 168-176, 2016.

3. Yu X, Wang Z, Shu Z, Li Z, Ning Y, Yun K, Bai H, Liu R and Liu W: Effect and mechanism of Sorbus pohuashanensis (Hante) Hedl. Flavonoids protect against arsenic trioxide-induced cardiotoxicity. Biomed Pharmacother 88: 1-10, 2017.

4. Raghu KG and Cherian OL: Characterization of cytotoxicity induced by arsenic trioxide (a potent anti-APL drug) in rat cardiac myocytes. J Trace Elem Med Biol 23: 61-68, 2009.

5. Varghese MV, Abhilash M, Paul MV, Alex M and Nair RH: Omega-3 fatty acid protects against arsenic trioxide-induced cardiotoxicity in vitro and in vivo. Cardiovasc Toxicol 17: 109-119, 2017.

6. Manna P, Sinha M and Sil PC: Arsenic-induced oxidative myocardial injury: Protective role of arjunolic acid. Arch Toxicol 82: 137-149, 2008.

7. Zhang W, Guo C, Gao R, Ge M, Zhu Y and Zhang Z: The protective role of resveratrol against arsenic trioxide-induced cardiotoxicity. Evid Based Complement Alternat Med 2013: 407839, 2013.

8. Abeyrathna P and Su Y: The critical role of Akt in cardiovascular function. Vascul Pharmacol 74: 38-48, 2015.

9. Wang Z, Yu J, Wu J, Qi F, Wang H, Wang Z and Xu Z: Scutellarin protects cardiomyocyte ischemia-reperfusion injury by reducing apoptosis and oxidative stress. Life Sci 157: 200-207, 2016.

10. Galano A: Free radicals induced oxidative stress at a molecular level: The current status, challenges and perspectives of computational chemistry based protocols. J Mex Chem Soc 59: 231-262, 2015.

11. Zhou SS, He F, Chen AH, Hao PY and Song XD: Suppression of rat frizzled-2 attenuates hypoxia/reoxygenation-induced $\mathrm{Ca}^{+}$ accumulation in rat H9c2 cells. Exp Cell Res 318: 1480-1491, 2012.

12. Andrades ME, Ritter $C$ and Dal-Pizzol F: The role of free radicals in sepsis development. Front Biosci (Elite Ed) 1: 277-287, 2009.

13. Baird AM, O'Byrne KJ and Gray SG: Reactive oxygen species and reactive nitrogen species in epigenetic modifications. Biochem Soc Trans: 437-455, 2012. 
14. Edson $\mathrm{KZ}$ and Rettie $\mathrm{AE}: \mathrm{CYP}_{4}$ enzymes as potential drug targets: Focus on enzyme multiplicity, inducers and inhibitors, and therapeutic modulation of 20-hydroxyeicosatetraenoic acid (20-HETE) synthase and fatty acid $\omega$-hydroxylase activities. Curr Top Med Chem 13: 1429-1440, 2013.

15. Nakayama H, Chen X, Baines CP, Klevitsky R, Zhang X, Zhang H, Jaleel N, Chua BH, Hewett TE, Robbins J, et al: $\mathrm{Ca}^{2+}-$ and Mitochondrial-dependent cardiomyocyte necrosis as a primary mediator of heart failure. J Clin Invest 117: 2431-2444, 2007.

16. Zhang J, Zhang Y, Wang W, Li C and Zhang Z: Double-sided personality: Effects of arsenic trioxide on inflammation. Inflammation 41: 1128-1134, 2018

17. Gasparini $\mathrm{C}$ and Feldmann M: NF- $\kappa \mathrm{B}$ as a target for modulating inflammatory responses. Curr Pharm Des 18: 5735-5745, 2012.

18. Li S, Wang Y, Zhao H, He Y and Xing M: NF- $\kappa B$-mediated inflammation correlates with calcium overload under arsenic trioxide-induced myocardial damage in Gallus gallus. Chemosphere 185: 618-627, 2017.

19. Saad SY, Alkharfy KM and Arafah MM: Cardiotoxic effects of arsenic trioxide/imatinib mesilate combination in rats. J Pharm Pharmacol 58: 567-573, 2006.

20. Sepand MR, Razavi-Azarkhiavi K, Omidi A, Zirak MR, Sabzevari S, Kazemi AR and Sabzevari O: Effect of acetyl-1-carnitine on antioxidant status, lipid peroxidation, and oxidative damage of arsenic in rat. Biol Trace Elem Res 171: 107-115, 2016.

21. Miao X, Tang Z, Wang Y, Su G, Sun W, Wei W, Li W, Miao L, Cai L, Tan Y and Liu Q: Metallothionein prevention of arsenic trioxide-induced cardiac cell death is associated with its inhibition of Mitogen-activated protein kinases activation in vitro and in vivo. Toxicol Lett 220: 277-285, 2013.

22. Zhao X, Feng T, Chen H, Shan H, Zhang Y, Lu Y and Yang B: Arsenic trioxide-induced apoptosis in $\mathrm{H} 9 \mathrm{c} 2$ cardiomyocytes: Implications in cardiotoxicity. Basic Clin Pharmacol Toxicol 102: 419-425, 2008

23. Testai L, Piragine E, Piano I, Flori L and Calderone V: The citrus flavonoid naringenin protects the myocardium from ageing-dependent dysfunction: Potential role of SIRT1. Oxid Med Cell Longev 2020: 4650207, 2020.

24. Pirola RC and Elmslie RG: Exchange transfusion and liver perfusion in the treatment of acute hepatic coma. Med J Aust 1: 891-894, 1968

25. Mu W, Zhang Q, Tang X, Fu W, Zheng W, Lu Y, Li H, Wei Y, Li L, She Z, et al: Overexpression of a dominant-negative mutant of SIRT1 in mouse heart causes cardiomyocyte apoptosis and Early-onset heart failure. Sci China Life Sci 57: 915-924, 2014.

26. Zhang W, Li Y and Ge Z: Cardiaprotective effect of crocetin by attenuating apoptosis in isoproterenol induced myocardial infarction rat model. Biomed Pharmacother 93: 376-382, 2017.

27. Batarseh YS, Bharate SS, Kumar V, Kumar A, Vishwakarma RA, Bharate SB and Kaddoumi A: Crocus sativus extract tightens the Blood-brain barrier, reduces amyloid beta load and related toxicity in 5XFAD mice. ACS Chem Neurosci 8: 1756-1766, 2017.

28. Hosseinzadeh H, Sadeghnia HR, Ziaee T and Danaee A: Protective effect of aqueous saffron extract (Crocus sativus L.) and crocin, its active constituent, on renal ischemia-reperfusion-induced oxidative damage in rats. J Pharm Pharm Sci 8: 387-393, 2005.

29. Umigai N, Murakami K, Ulit MV, Antonio LS, Shirotori M, Morikawa $\mathrm{H}$ and Nakano T: The pharmacokinetic profile of crocetin in healthy adult human volunteers after a single oral administration. Phytomedicine 18: 575-578, 2011.

30. Gao K, Liu F, Chen X, Chen M, Deng Q, Zou X and Guo H: Crocetin protects against fulminant hepatic failure induced by lipopolysaccharide/D-galactosamine by decreasing apoptosis, inflammation and oxidative stress in a rat model. Exp Ther Med 18: 3775-3782, 2019.

31. Bolhassani A, Khavari A and Bathaie SZ: Saffron and natural carotenoids: Biochemical activities and anti-tumor effects. Biochim Biophys Acta 1845: 20-30, 2014.

32. Shen XC and Qian ZY: Effects of crocetin on antioxidant enzymatic activities in cardiac hypertrophy induced by norepinephrine in rats. Pharmazie 61: 348-352, 2006.

33. Yang L, Qian Z, Ji H, Yang R, Wang Y, Xi L, Sheng L, Zhao B and Zhang $\mathrm{X}$ : Inhibitory effect on protein kinase Ctheta by Crocetin attenuates palmitate-induced insulin insensitivity in 3T3-L1 adipocytes. Eur J Pharmacol 642: 47-55, 2010.
34. Diao SL, Sun JW, Ma BX, Li XM and Wang D: Influence of crocetin on high-cholesterol diet induced atherosclerosis in rats via anti-oxidant activity together with inhibition of inflammatory response and p38 MAPK signaling pathway. Saudi J Biol Sci 25: 493-499, 2018

35. Mancini A, Serrano-Díaz J, Nava E, D'Alessandro AM, Alonso GL, Carmona M and Llorens S: Crocetin, a carotenoid derived from saffron (Crocus sativus L.), improves acetylcholine-induced vascular relaxation in hypertension. J Vasc Res 51: 393-404, 2014.

36. Cai J, Yi FF, Bian ZY, Shen DF, Yang L, Yan L, Tang QZ, Yang $\mathrm{XC}$ and $\mathrm{Li} \mathrm{H}$ : Crocetin protects against cardiac hypertrophy by blocking MEK-ERK1/2 signalling pathway. J Cell Mol Med 13: 909-925, 2009.

37. Shen XC and Qian ZY: Effect of crocetin on cardiac hypertrophy induced by overloading pressure in rats. Acta Pharm Sin 39: 172-175, 2004.

38. Li CY, Huang WF, Wang QL, Wang F, Cai E, Hu B, Du JC, Wang J, Chen R, Cai XJ, et al: Crocetin induces cytotoxicity in colon cancer cells via p53-independent mechanisms. Asian Pac J Cancer Prev 13: 3757-3761, 2012.

39. Kim SH, Lee JM, Kim SC, Park CB and Lee PC: Proposed cytotoxic mechanisms of the saffron carotenoids crocin and crocetin on cancer cell lines. Biochem Cell Biol 92: 105-111, 2014.

40. Yang M, Mao G, Ouyang L, Shi C, Hu P and Huang S: Crocetin alleviates myocardial ischemia/reperfusion injury by regulating inflammation and the unfolded protein response. Mol Med Rep 21: 641-648, 2020.

41. Zhang D: Comparison of the effect of different anesthetics in five hundred rats. Lab Anim Sci 1: 19-20, 2007.

42. Zhao H, Haitao LI, Dingwei GU, Suxin LI and Fang Y: Effects of oral spironolactone on TNF- $\alpha$ expression of myocardial tissues in spontaneous hypertension rats. Tianjin Med J 40: 64-66, 2012.

43. Wang T, Sun X, Cui H, Liu K and Zhao J: The peptide compound urantide regulates collagen metabolism in atherosclerotic rat hearts and inhibits the JAK2/STAT3 pathway. Mol Med Rep 21: 1097-1106, 2020

44. Knuckles TL, Buntz JG, Paffett M, Channell M, Harmon M, Cherng T, Lucas SN, McDonald JD, Kanagy NL and Campen MJ: Formation of vascular s-nitrosothiols and plasma nitrates/nitrites following inhalation of diesel emissions. J Toxicol Environ Health A 74: 828-837, 2011.

45. Song Q, Chu X, Zhang X, Bao Y, Zhang Y, Guo H, Liu Y, Liu H, Zhang J, Zhang Y and Chu L: Mechanisms underlying the cardioprotective effect of Salvianic acid A against isoproterenol-induced myocardial ischemia injury in rats: Possible involvement of L-type calcium channels and myocardial contractility. J Ethnopharmacol 189: 157-164, 2016.

46. Han X, Li M, Zhao Z, Zhang Y, Zhang J, Zhang X, Zhang Y, Guan S and Chu L: Mechanisms underlying the cardio-protection of total ginsenosides against myocardial ischemia in rats in vivo and in vitro: Possible involvement of $\mathrm{L}$-type $\mathrm{Ca}^{2+}$ channels, contractility and $\mathrm{Ca}^{2+}$ homeostasis. J Pharmacol Sci 139: 240-248, 2019.

47. Lee T, Chen $C$ and Chang N: Cardiac sympathetic hyperinnervation in deoxycorticosterone acetate-salt hypertensive rats. Clin Sci (Lond) 123: 445-457, 2012.

48. Li R, Liu Y, Xie J, Huang X, Zhang L, Liu H and Li L: Sirt3 mediates the protective effect of hydrogen in inhibiting ROS-induced retinal senescence. Free Radic Biol Med 135: 116-124, 2019.

49. Jin W, Xue Y, Xue Y, Han X, Song Q, Zhang J, Li Z, Cheng J, Guan S, Sun S and Chu L: Tannic acid ameliorates arsenic Trioxide-induced nephrotoxicity, contribution of NF- $\kappa \mathrm{B}$ and Nrf2 pathways. Biomed. Pharmacother 126: 110047, 2020.

50. Meister AL, Doheny KK and Travagli RA: Necrotizing enterocolitis attenuates developmental heart rate variability increases in newborn rats. Neurogastroenterol Motil 31: e13484, 2019.

51. Jin W, Zhang Y, Xue Y, Han X, Zhang X, Ma Z, Sun S, Chu X, Cheng J, Guan S, et al: Crocin attenuates isoprenaline-induced myocardial fibrosis by targeting TLR $4 / N F-\kappa B$ signaling: Connecting oxidative stress, inflammation, and apoptosis. Naunyn Schmiedebergs Arch Pharmacol 393: 13-23, 2020.

52. Chu L, Li P, Song T, Han X, Zhang X, Song Q, Liu T, Zhang Y and Zhang J: Protective effects of tannic acid on pressure overload-induced cardiac hypertrophy and underlying mechanisms in rats. J Pharm Pharmacol 69: 1191-1207, 2017.

53. Sun Q, Jia N, Wang W, Jin H, Xu J and Hu H: Activation of SIRT1 by curcumin blocks the neurotoxicity of amyloid- $\beta$ 25-35 in rat cortical neurons. Biochem Biophys Res Commun 448: 89-94, 2014. 
54. Tallman MS: Treatment of relapsed or refractory acute promyelocytic leukemia. Best Pract Res Clin Haematol 20: 57-65, 2007.

55. Sun TL, Liu Z, Qi ZJ, Huang YP, Gao XQ and Zhang YY: (-)-Epigallocatechin-3-gallate (EGCG) attenuates arsenic-induced cardiotoxicity in rats. Food Chem Toxicol 93 102-110, 2016.

56. Hashemi M and Hosseinzadeh $\mathrm{H}$ : A comprehensive review on biological activities and toxicology of crocetin. Food Chem Toxicol 130: 44-60, 2019.

57. Liang X and Qian Z: Pharmacological properties of crocetin and crocin (digentiobiosyl ester of crocetin) from saffron. Nat Prod Commun 1: 65-75, 2006.

58. Hosseini A, Razavi BM and Hosseinzadeh H: Pharmacokinetic properties of saffron and its active components. Eur J Drug Metab Pharmacokinet 43: 383-390, 2018.

59. Xi L, Qian Z, Du P and Fu J: Pharmacokinetic properties of crocin (crocetin digentiobiose ester) following oral administration in rats. Phytomedicine 14: 633-636, 2007.

60. Asai A, Nakano T, Takahashi M and Nagao A: Orally administered crocetin and crocins are absorbed into blood plasma as crocetin and its glucuronide conjugates in mice. J Agric Food Chem 53: 7302-7306, 2005 .

61. Zhang Y, Fei F, Zhen L, Zhu X, Wang J, Li S, Geng J, Sun R, $\mathrm{Yu} \mathrm{X}$, Chen T, et al: Sensitive analysis and simultaneous assessment of pharmacokinetic properties of crocin and crocetin after oral administration in rats. J Chromatogr B Analyt Technol Biomed Life Sci 1044-1045: 1-7, 2017.

62. Farkhondeh T, Samarghandian S, Samini F and Sanati AR: Protective effects of crocetin on depression-like behavior induced by immobilization in rat. CNS Neurol Disord Drug Targets 17: 361-369, 2018.

63. Danesi R, Tacca MD and Soldani G: Measurement of the SaT segment as the most reliable electrocardiogram parameter for the assessment of adriamycin-induced cardiotoxicity in the rat J Pharmacol Methods 16: 251-259, 1986.

64. Kumari P, Saifi MA, Khurana A and Godugu C: Cardioprotective effects of nanoceria in a murine model of cardiac remodeling. J Trace Elem Med Biol 50: 198-208, 2018.

65. Tang Y, Wang M, Le X, Meng J, Huang L, Yu P, Chen J and Wu P: Antioxidant and cardioprotective effects of Danshensu (3-(3,4-dihydroxyphenyl)-2-hydroxy-propanoic acid from Salvia miltiorrhiza) on isoproterenol-induced myocardial hypertrophy in rats. Phytomedicine 18: 1024-1030, 2011.

66. Shan H, Zhang Y, Cai B, Chen X, Fan Y, Yang L, Chen X, Liang H, Zhang Y, Song X, et al: Upregulation of microRNA-1 and microRNA-133 contributes to arsenic-induced cardiac electrical remodeling. Int J Cardiol 167: 2798-2805, 2013.

67. Drolet B, Simard C and Roden DM: Unusual effects of a QT-prolonging drug, arsenic trioxide, on cardiac potassium currents. Circulation 109: 26-29, 2004.

68. Aposhian HV and Aposhian MM: Arsenic toxicology: Five questions. Chem Res Toxicol 19: 1-15, 2006.

69. Hwang JT, Kwon DY, Park OJ and Kim MS: Resveratrol protects ROS-induced cell death by activating AMPK in H9c2 cardiac muscle cells. Genes Nutr 2: 323-326, 2008

70. Oudit GY, Trivieri MG, Khaper N, Husain T, Wilson GJ, Liu P, Sole MJ and Backx PH: Taurine supplementation reduces oxidative stress and improves cardiovascular function in an Iron-overload murine model. Circulation 109: 1877-1885, 2004.
71. Li SW, Sun X, He Y, Guo Y, Zhao HJ, Hou ZJ and Xing MW: Assessment of arsenic trioxide in the heart of Gallus gallus: Alterations of oxidative damage parameters, inflammatory cytokines, and cardiac enzymes. Environ Sci Pollut Res Int 24: 5781-5790, 2017.

72. Kowalczuk K and Stryjecka-Zimmer M: The influence of oxidative stress on the level of malondialdehyde (MDA) in different areas of the rabbit brain. Ann Univ Mariae Curie Sklodowska Med 57: 160-164, 2002.

73. Djordjevic A, Spasic S, Jovanovic-Galovic A, Djordjevic R and Grubor-Lajsic G: Oxidative stress in diabetic pregnancy: SOD, CAT and GSH-Px activity and lipid peroxidation products. J Matern Fetal Neonatal Med 16: 367-372, 2004.

74. Alexa ID and Jerca L: The role of oxidative stress in the etiology of Pre-eclampsia: Changes at the GSH and GSH-Px levels in normal pregnancy and pre-eclampsia. Rev Med Chir Soc Med Nat Iasi 100: 131-135, 1996 (In Romanian).

75. Espinosa J, Pérez JM, López-Olvera JR, Ráez-Bravo A, Cano-Manuel FJ, Fandos P, Soriguer RC, Granados JE and Romero D: Evaluation of oxidant/antioxidant balance in Iberian ibex (Capra pyrenaica) experimentally infested with Sarcoptes scabiei. Vet Parasitol 242: 63-70, 2017.

76. Yang R, Yang L, Shen X, Cheng W, Zhao B, Ali KH, Qian Z and Ji H: Suppression of NF- $\mathrm{BB}$ pathway by crocetin contributes to attenuation of lipopolysaccharide-induced acute lung injury in mice. Eur J Pharmacol 674: 391-396, 2012.

77. Wang Y, Yu W, Shi C and Hu P: Crocetin attenuates sepsisinduced cardiac dysfunction regulation of inflammatory response and mitochondrial function. Front Physiol 11: 514, 2020.

78. Song L, Kang C, Sun Y, Huang WR, Liu W and Qian ZY: Crocetin inhibits lipopolysaccharide-induced inflammatory response in human umbilical vein endothelial cells. Cell Physiol Biochem 40: 443-452, 2016.

79. Mantawy EM, El-Bakly WM, Esmat A, Badr AM and El-Demerdash E: Chrysin alleviates acute doxorubicin cardiotoxicity in rats via suppression of oxidative stress, inflammation and apoptosis. Eur J Pharmacol 728: 107-118, 2014.

80. Liu MH, Shan J, Li J, Zhang Y and Lin XL: Resveratrol inhibits Doxorubicin-induced cardiotoxicity via sirtuin 1 activation in H9c2 cardiomyocytes. Exp Ther Med 12: 1113-1118, 2016.

81. Sun Z, Lu W, Lin N, Lin H, Zhang J, Ni T, Meng L, Zhang C and Guo H: Dihydromyricetin alleviates doxorubicin-induced cardiotoxicity by inhibiting NLRP3 inflammasome through activation of SIRT1. Biochem Pharmacol 175: 113888, 2020.

82. Binu P, Priya N, Abhilash S, Vineetha R and Nair R: Studies on curative efficacy of monoterpene eugenol on anti-leukemic drug arsenic trioxide induced cardiotoxicity. Biomed Pharmacother 91: 559-566, 2017.

This work is licensed under a Creative Commons Attribution-NonCommercial-NoDerivatives 4.0 International (CC BY-NC-ND 4.0) License. 